APH N.S., Heavy Ion Physics 5 (1997) 319-320

\title{
Erratum: Complete Spin Structure of the Pion-Nucleon- Loop Delta Self-Energy
}

\author{
C.L. Korpa
}

Department of Theoretical Physics, Janus Pannonius University Ifjúság útja 6, 7624 Pécs, Hungary

Received 14 May 1997

Abstract. A calculational error is corrected, numerical results are affected very little.

In Ref. [1] a sign error in an intermediate result led to erroneous expressions for some of the terms $\alpha_{i}\left(p^{2}\right)$ in expression ( 9 ). The corrected (including also typographical errors) expressions are the following:

$$
\begin{aligned}
\alpha_{1} & =-\frac{M k_{*}^{2}}{3} \\
\alpha_{2} & =-\frac{k_{*}^{2}}{6 p^{2}}\left(p^{2}+M^{2}-m^{2}\right) \\
\alpha_{5} & =-\frac{a M}{3}\left(2 k_{*}^{2}-3 a m^{2}\right) \\
\alpha_{6} & =-\frac{2}{3} a k_{*}^{2}-\frac{a^{2}}{2 p^{2}}\left[p_{*}^{4}-m^{2}\left(3 p^{2}-M^{2}+m^{2}\right)\right] \\
\alpha_{9} & =\frac{1}{6 p^{2}}\left[k_{*}^{2} p_{*}^{2}+a\left(p_{*}^{4}+2 m^{2} p^{2}-3 m^{2} p_{*}^{2}\right)\right] \\
\alpha_{10} & =\frac{1}{6 p^{2}}\left\{k_{*}^{2} p_{*}^{2}+3 a(1+2 a)\left[p_{*}^{4}-2 m^{2} p^{2}-m^{2} p_{*}^{2}\right]\right\}
\end{aligned}
$$

There were also typographical overall sign errors on the right-hand sides of expressions (3), (5) and (7).

Numerical results are affected very little by the corrections. The coupling and the cut-off are slightly larger than quoted in [1], for example an excellent fit to spin-3/2 isospin-3/2 pion-nucleon phase shift has been achieved with $g=19$, $\Lambda=0.97 \mathrm{GeV}, M_{\Delta}=1.279 \mathrm{GeV}, a=-0.5$. A somewhat larger coupling 
( $g=20)$ and smaller cut-off $(\Lambda=0.92 \mathrm{GeV})$ also gives an excellent fit (with $M_{\Delta}=1.273 \mathrm{GeV}$ ). The off-shell parameter $a$ can take values between -1 and 0 without affecting significantly the phase shift.

\section{References}

1. C.L. Korpa, Heavy Ion Phys. 5 (1997) 77. 